\title{
Médiévales
}

Langues, Textes, Histoire

58 | printemps 2010

Humanisme et découvertes géographiques

\section{L'histoire toute crue : la Première Croisade au miroir de son Histoire}

Damien Kempf et Marcus Bull

\section{(2) OpenEdition}

1 Journals

Édition électronique

URL : https://journals.openedition.org/medievales/6017

DOI : 10.4000/medievales.6017

ISSN : $1777-5892$

Éditeur

Presses universitaires de Vincennes

Édition imprimée

Date de publication : 30 juin 2010

Pagination : 151-160

ISBN : 978-2-84292-260-3

ISSN : 0751-2708

Référence électronique

Damien Kempf et Marcus Bull, «L'histoire toute crue : la Première Croisade au miroir de son Histoire », Médiévales [En ligne], 58 I printemps 2010, mis en ligne le 20 septembre 2012, consulté le 23 avril 2022. URL : http://journals.openedition.org/medievales/6017 ; DOI : https://doi.org/10.4000/ medievales.6017 
Médiévales 58, printemps 2010, p. 151-160

Damien KEMPF et Marcus BULL

\section{L'HISTOIRE TOUTE CRUE : LA PREMIÈRE CROISADE AU MIROIR DE SON HISTOIRE}

L'essence pure du récit tel qu'on peut idéalement le concevoir, c'est l'histoire toute crue, la série intacte des événements tels qu'ils sont apparus dans le monde, fût-il fictif, avant que ne vienne s'en emparer la parole du narrateur.

Gérard GenetTe, Figures II, Paris, 1972, p. 63.

L'Histoire des Croisades ne s'est jamais aussi bien portée, en particulier dans les pays anglo-saxons : on ne compte plus les «Histoires », « Histoires Brèves », « Nouvelles Histoires » des Croisades, qui toutes revendiquent une nouvelle approche, une interprétation différente, un point de vue unique sur la période ${ }^{1}$. Plutôt que des écrits destinés uniquement à des spécialistes, où l'érudition se mêlerait à la diatribe, nombre de ces ouvrages s'adressent aussi au grand public, ou à tout le moins entendent toucher un lectorat qui ne soit pas exclusivement universitaire. Le temps n'est pas à la démonstration savante et fastidieuse, aux notes de bas de page innombrables. Le temps est bien au récit, à l'anecdote. On ne disserte guère, on raconte. On retrace les aventures extraordinaires de ceux qui, à plusieurs reprises, partirent pour la Terre Sainte au nom d'idéaux chrétiens. On suit, presque au jour le jour, les faits et gestes de tous ces « chevaliers d'aventure » qui ont fait des Croisades un thème populaire, une odyssée religieuse, voire un refrain nationaliste. Même quand l'entreprise se veut moins narrative, et plus thématique, elle entend être au plus près des aspirations, des émotions, des expériences des acteurs des Croisades : comme le dit la jaquette d'un ouvrage récent, « on entend les épées, on goûte la nourriture, on voit les sites, et on ressent la chaleur $»$.

Ce penchant narratif, qui a caractérisé l'Histoire des Croisades depuis qu'elle s'est solidement constituée en discipline (pensons à la grande synthèse toujours inégalée de Steven Runciman) ${ }^{2}$, reflète la nature même des sources,

1. Citons, entre autres : J. Phillips, Holy Warriors : A Modern History of the Crusades, Londres, 2009 ; ID., The Second Crusade : Extending the Frontiers of Christendom, Yale, 2007 ; C. Tyerman, God's War : A New History of the Crusades, Londres, 2007 ; T. Asbridge, The First Crusade : A New History, Oxford, 2004.

2. S. Runciman, A History of the Crusades, 3 vol., Cambridge, 1951-1954. 
dont beaucoup sont narratives. Cela est particulièrement vrai pour la Première Croisade, qui va nous occuper ici. Comme le notait déjà l'un des pionniers de l'historiographie moderne des Croisades, Heinrich von Sybel, qui publiait en 1841 une Histoire de la Première Croisade, en plus d'être un événement historique majeur, la Première Croisade fut aussi un événement historiographique : il est difficile de trouver un autre fait au Moyen Âge qui ait provoqué la composition d'autant de sources de nature diverse, et en particulier de récits ${ }^{3}$. Témoignages oculaires, écrits par des participants aux événements, ou chroniques rédigées dans les années qui suivirent la prise de Jérusalem en 1099, c'est au bas mot une douzaine de récits que nous possédons ${ }^{4}$. Sybel pouvait ainsi remarquer : «Nous avons une telle quantité de sources qu'on devrait penser que nous pouvons rétablir pour toujours le déroulement des événements. En effet, au moment où l'on commence à se familiariser avec tous ces récits et discours, on ne cesse de s'étonner de la vie qui transparaît au travers de ces textes ; dès le début, on a l'espoir de pénétrer jusqu'aux faits et de saisir à l'intérieur le noyau ${ }^{5} »$.

3. H. vON Sybel, Geschichte des ersten Kreuzzugs, Düsseldorf, 1841, p. 3.

4. Les témoignages oculaires sont les suivants : Gesta Francorum, dont Marcus Bull prépare une nouvelle édition, qui sera publiée par Oxford University Press en 2011 (dans la collection «Oxford Medieval Texts »). En attendant, on peut se reporter aux éditions d'Heinrich HAGENMEYER (Anonymi Gesta Francorum et aliorum Hierosolimitanorum, Heidelberg, 1890), de Rosalind HiLL (Gesta Francorum et aliorum Hierosolimitanorum, Londres, 1962), et de Louis BRÉHIER (Histoire anonyme de la Première Croisade, Paris, 1924) ; Foucher DE Chartres, Fulcheri Carnotensis Historia Hierosolymitana (1095-1127), H. HagenMeYer éd., Heidelberg, 1913 ; Pierre Tudebode, Historia de Hierosolymitano itinere, J. H. Hill et L. L. Hill éd., Paris, 1977 ; RaYmond d'Aguilers, Historia Francorum qui ceperunt Iherusalem, J. H. Hill et L. L. Hill éd., Philadelphie, 1968. Parmi les récits ultérieurs, mentionnons : RaOul DE CAEN, Gesta Tancredi inexpeditione Hierosolymitana ...auctore Radulfo Cadomensi, dans Recueil des Historiens des Croisades, Historiens Occidentaux (désormais abrégé en : RHC Occ.), t. 3, p. 587-716 ; GuIBERT DE Nogent, Dei gesta per Francos, R. B.C. HuYgens éd., Turnhout, 1996 (Corpus Christianorum, Continuatio Mediaevalis, 127A) ; trad. fr. M.-C. GaRAnd, Guibert de Nogent, Geste de Dieu par les Francs: Histoire de la Première Croisade, Turnhout, 1998; Albert D'Aix-la-Chapelle, Historia Ierosolimitana. History of the Journey to Jerusalem, S. B. Edgington éd. et trad., Oxford, 2007 ; BAUdRI De BourgueIL, Baldrici episcopi Dolensis Historia Jerosolimitana, dans RHC Occ., t. 4, p. 1-111 ; EKKEHARD D'AURA, Ekkehardi abbatis Uraugiensis Hierosolymita, dans RHC Occ., t. 5, p. 1-40 ; RoBerT Le MoINe, Roberti Monachi historia Iherosolimitana, dans RHC Occ., t. 3, p. 717-882; Guillaume De Tyr, Chronique, R. B. C. Huygens éd., 2 vols, Turnhout, 1986 (Corpus Christianorum, Continuatio Mediaevalis, 63-63A) ; La chronique anonyme de Monte Cassino, ou Historia peregrinorum euntium Jerusolymam, dans RHC Occ., t. 3, p. 167-229. Pour une vue d'ensemble de la production, on peut se référer à S. B. EdGington, «The First Crusade: Reviewing the evidence», dans J. P. Phillips dir., The First Crusade : Origins and Impact, Manchester, 1996, p. 57-77 ; R. HiestAND, « Il cronista medievale el il suo pubblico : alcune osservazioni in margine alla storiografia delle crociate », Annali della Facoltà di Lettere e Filosofia dell'Università di Napoli, t. 27, 1984-85, p. 207-227.

5. H. vON SYBEL, op. cit., p. 3. 
Il est vrai que la nature de la documentation et sa richesse permettent de faire pour la Première Croisade ce qu'il est souvent difficile de réaliser pour le Moyen Âge : suivre pas à pas les croisés, leur itinéraire, les lieux et villes qui ont jalonné leur parcours. C'est une véritable quotidienneté de l'expédition qui surgit au travers de la lecture des sources. C'est précisément cette quotidienneté que la plupart des chroniqueurs médiévaux, qu'ils aient ou non participé à l'expédition, tentèrent d'approcher au plus près.Etc'est cette même quotidienneté que les historiens des Croisades, depuis Sybel, ont tenté, dans un réflexe mimétique, de reconstituer. $\mathrm{La}$ « Chronologie de la première croisade » publiée par Heinrich Hagenmeyer en 1902 incarne parfaitement cette approche « recréationniste » : sur la base principalement des sources narratives, l'érudit allemand retraça le calendrier des actions menées par les croisés, au jour le jour, établissant ainsi un véritable journal de marche ${ }^{6}$.

Les sources relatives à cette période sont encore trop souvent traitées, en effet, comme une simple documentation, comme réservoir d'informations permettant de restituer, aussi précisément que possible, le déroulement des événements. C'est en se constituant en discipline à part que l'Histoire des Croisades s'est figée en histoire événementielle «traités-et-batailles » dans la droite ligne des travaux et aspirations de Leopold von Ranke ${ }^{7}$. Ainsi l'Histoire des Croisades n'a-t-elle guère évolué au gré des différents courants qui ont traversé et caractérisé le champ historiographique de ces cinquante dernières années. Pas d'école des Annales, pas de structuralisme, encore moins de linguistic turn ${ }^{8}$. Cet isolement académique a entraîné ce que nous pourrions appeler une ségrégation des sources : tout ce qui ressortait de l'histoire des Croisades devint le domaine réservé des spécialistes de la période. Le répertoire extrêmement riche et varié des sources a ainsi eu tendance à être considéré comme un genre en tant que tel, comme si tout document relatif aux croisades appartenait, par définition, et par essence, à l'Histoire des Croisades érigée en discipline. Du coup, et pour s'en tenir aux sources qui nous intéressent ici en premier lieu, les sources narratives, les nombreux récits qui portent sur la Première Croisade, et qui font de cet événement un champ unique dans la production historiographique médiévale, n'ont guère été étudiés par les spécialistes de l'historiographie. Comme le remarquait très justement Giles Constable, «il est intéressant, et peut-être significatif, qu'il n'y ait pas de traitement soutenu de l'historiographie dans

6. H. Hagenmeyer, Chronologie de la première croisade (1094-1100), Paris, 1902.

7. Pour une analyse de l'œuvre et des idées de Ranke, on pourra se reporter à W. J. Mommsen dir., Leopold von Ranke und die moderne Geschichtswissenschaft, Stuttgart, 1999.

8. Une bonne synthèse des principes méthodologiques et des enjeux du linguistic turn pour les historiens se trouve dans E. CLARK, History, Theory, Text : Historians and the Linguistic Turn, Cambridge, Mass., 2005. 
les histoires générales des Croisades par René Grousset, Steven Runciman et Hans Eberhard Meyer ${ }^{9} »$.

Tout comme leurs prédécesseurs médiévaux sur lesquels ils s’appuient, les historiens modernes ont fondé leur discipline autour d'une conviction majeure, celle de «pénétrer jusqu'aux faits » comme le souhaitait Sybel. Tout comme leurs prédécesseurs médiévaux, ils n'ont eu de cesse d'écrire, de réécrire l'histoire de ces hommes qui décidèrent de participer à la libération de Jérusalem. Avec, pour point de départ, un récit qui est souvent présenté comme le premier témoignage oculaire de l'expédition, le plus authentique, le plus fiable, les Gesta Francorum et aliorum Hierosolimitanorum. Comme nous voudrions le montrer, c'est en perpétuant un mythe d'origine médiévale autour des Gesta que l'historiographie moderne des Croisades, dans sa volonté d'approcher au plus près les faits, s'est forgée sa légitimité : en faisant des Gesta un récit «pur», un récit qui livrerait les événements tels qu'ils se sont déroulés, sans la parole filtrante de l'auteur, les historiens se sont donné la possibilité de continuer à tisser la trame d'un récit fondé sur l'ambition de restituer « la série intacte des événements tels qu'ils sont apparus dans le monde ${ }^{10} »$.

Mais il est sans doute bon de commencer par dire quelques mots sur la Première Croisade elle-même. Lancée par le pape Urbain II à Clermont-Ferrand en novembre 1095, la croisade fut une série convergente d'expéditions parties d'Occident vers la Palestine afin de libérer Jérusalem de la domination turque. Différentes troupes se mirent en marche durant l'année 1096, certaines se regroupant dans le Nord-Ouest de la Turquie moderne avant d'entamer une ardente campagne de deux ans, dont l'apogée fut la prise de la Ville Sainte, en juillet 1099. Le nombre de croisés, clercs et soldats confondus, est très difficile à estimer, mais il pourrait avoir atteint cent mille hommes. Après la conquête de Jérusalem, la plupart des soldats rentrèrent chez eux, laissant derrière eux quelques centaines de congénères qui prirent en main l'administration de la ville. Ce fut le début d'un processus récurrent de campagnes militaires qui se déroulèrent tout au long des décennies et siècles suivants, un cycle de croisades qui furent toutes menées sur le modèle de celle qui était maintenant appelée la « Première » Croisade, dont l'éclatant succès continua d'inspirer et d'alimenter les aspirations guerrières de nombreuses générations.

C'est à n'en pas douter la nature et l'issue extraordinaires de la Première Croisade qui poussèrent de nombreux auteurs à prendre leur plume pour célébrer les exploits de ceux qui permirent la conquête (en fait, une

9. G. Constable, « The Historiography of the Crusades », dans A. E. Laiou et R. Parviz Mottahedeh dir., The Crusades from the Perspective of Byzantium and the Muslim World, Washington D.C., 2001, p. 2.

10. G. Genette, Figures II, Paris, 1972, p. 63. 
« reconquête » dans l'esprit de ses instigateurs) de Jérusalem, et d'en faire une capitale chrétienne. Le spécialiste a donc devant lui une mine d'informations, de détails qui feraient pâlir la plupart de ses collègues. Ce sont non seulement les acteurs, mais aussi leurs faits d'armes, les lieux, qui nous sont ainsi livrés dans une chronologie très fine. Tout commença, pour ainsi dire, avec les Gesta Francorum ${ }^{11}$.

Racontée par un auteur anonyme, l'œuvre est un récit en prose de près de vingt mille mots, dont le titre, à défaut d'être original, apparaît dans les manuscrits les plus anciens. Contrairement à d'autres récits, les Gesta ne s'ouvrent pas par le sermon d'Urbain II, mais débutent par la tournée du pape en France. Le récit s'attache d'abord principalement à Bohémond de Tarente, un aristocrate normand d'Italie du Sud, qui fut l'un des leaders de la Première Croisade. Le lecteur suit ses pérégrinations d'Italie jusqu'en Asie Mineure, pour arriver à la prise d'Antioche en juin 1098, qui marque l'un des tournants majeurs de l'expédition. L'auteur, qui ne mentionne pas que Bohémond choisit de rester à Antioche, au début de l'année 1099, suit alors le long périple qui va conduire les troupes de Raymond de Saint Gilles, un autre personnage important de la Première Croisade, vers Jérusalem. Les Gesta s'achèvent, de façon plutôt abrupte, par la victoire des croisés contre les troupes égyptiennes à Ascalon (au nord de la Bande de Gaza), le 12 août 1099. Il est, par conséquent, fort vraisemblable que ce soit le dernier événement auquel l'auteur ait participé, avant de mettre un terme à son récit.

L'impact des Gesta fut considérable et immédiat : il servit de base aux autres témoignages oculaires : ceux de Foucher de Chartres, de Raymond d'Aguilers, voire de Pierre Tudebode, encore que ce dernier point soit l'objet de nombreux débats dont nous reparlerons plus loin. Le texte fut aussi repris, dès la première décennie du XII ${ }^{\mathrm{e}}$ siècle, par un groupe de moines du nord de la France, que Riley-Smith a pertinemment appelé le « groupe des théologiens ${ }^{12}$ », à savoir Guibert de Nogent, Robert le Moine et Baudri de Bourgueil. Chacun des trois auteurs se proposa de «corriger» le style défaillant des Gesta. Pour Robert, le texte est «confus dans son style et expression ${ }^{13} »$; c'est un récit

11. La littérature est nombreuse : signalons, entre autres, J. France, « The Use of the Anonymous Gesta Francorum in the Early Twelfth-Century Sources for the First Crusade », dans A. V. Murray dir., From Clermont to Jerusalem: The Crusades and Crusader Societies 1095-1500, Selected Proceedings of the International Medieval Congress, University of Leeds, 10-13 July 1995, Turnhout, 1998, p. 29-42 ; J. RubENSTEIN, «What is the Gesta Francorum, and who was Peter Tudebode? », Revue Mabillon, t. 16, 2005, p. 179-204 ; K. B. Wolf, «Crusade and Narrative : Bohemond and the Gesta Francorum», Journal of Medieval History, t. 17, 1991, p. 207-216; C. Morris, «The Gesta Francorum as Narrative History », Reading Medieval Studies, t. 19, 1993, p. 55-71.

12. J. Riley-Smith, The First Crusade and the Idea of Crusading, Londres, 1986, p. 135-52.

13. « litteralium compositio dictionum incondita » (RHC Occ, t. 3, p. 721). 
« extrêmement grossier » selon Baudri ${ }^{14}$, rédigé « dans un style plus que plat, avec des mots ordinaires ${ }^{15} \gg$ (Guibert).

Mais c'est cette apparente simplicité, cette prose « insipide », « toujours à ras de terre ${ }^{16} »$, qui fit précisément des Gesta une source digne de confiance aux yeux de nos moines théologiens. Si les trois auteurs se sont, en effet, servis de cette chronique comme modèle pour construire leur récit, tant au niveau des informations que dans la structure narrative, c'est parce qu'ils considéraient que l'auteur anonyme avait rapporté les faits « avec un grand souci de vérité », comme l'admet Guibert ${ }^{17}$. De par leur style et leur syntaxe élémentaires, leur vocabulaire réduit, les Gesta acquéraient une valeur toute particulière, celle d'un témoignage authentique et véridique. Et c'est en ce sens qu'il faut comprendre la critique acerbe, qui équivaut purement et simplement à un rejet, du récit de Foucher, pourtant lui aussi un témoin oculaire des événements, par Guibert. Ce dernier n'a pas de mots assez durs pour caractériser ce texte qu'il qualifie de «faux», au «style pompeux », émaillé de «mots ampoulés ${ }^{18}$. Pour Guibert donc, comme pour Robert et Baudri, c'est le style rudimentaire des Gesta qui leur assure une place prééminente. Autrement dit, c'est dans sa propre faiblesse que réside la principale qualité du texte, sa vérité.

C'est le point de départ d'un mythe qui sera repris verbatim par l'historiographie moderne, et cela dès Sybel. Pour ce dernier, comme pour Heinrich Hagenmeyer, qui établit la première sérieuse édition critique des Gesta en $1890^{19}$, la caractéristique principale, donc la qualité première du texte, c'est sa simplicité naïve. Le récit est interprété comme un Tagebuch, un journal intime : l'absence complète de remarques rétrospectives ou de réflexions tout au long du texte laisse supposer qu'il fut composé par à-coups, au fur et à mesure des événements, ce que suggère aussi sa fin quelque peu brusque, sa non-clôture.

14. « sed nescio quis compilator, nomine suppresso, libellum super hac re nimis rusticanum ediderat » (RHC Occ, t. 4, p. 10).

15. «Erat siquidem eadem Historia, sed verbis contexta plus equo simplicibus et quae multotiens grammaticae naturas excederet lectoremque vapidi insipidate sermonis sepius exanimare valeret» (Guibert de Nogent, Dei gesta per Francos, R. B. C. Huygens éd., op. cit., p. 79 ; trad. fr. M.-C. Garand, op. cit., p. 45).

16. Guibert encore, faisant ici une référence savante à l'Art poétique d'Horace.

17. «Quorum tenorem ab illo priori quem prosequor auctore multa veritate prolatum ab eis qui eidem interfuerant viae edidici, ea sane quae ferebantur in libro contuli crebrius cum ipsorum qui facta viderant verbo, et proculdubio expertus sum quia neutrum discreparet ab latero » (R. B. C. Huygens éd., op. cit., p. 82 ; trad. fr., M.-C. Garand, op. cit., p. 47).

18. R. B. C. Huygens éd., op. cit., p. 329 ; trad. fr. M.-C. Garand, op. cit., p. 285.

19. Les responsables du Recueil des Historiens Occidentaux, qui se montrèrent, de façon générale, bien peu soigneux dans leur labeur philologique, se contentant la plupart du temps d'amalgamer des variantes trouvées dans différents manuscrits, pensaient que les Gesta dérivaient du texte de Pierre Tudebode, d'où le titre trompeur de Gesta Francorum et aliorum Hierosolymitanorum, seu Tudebodus Abbreviatus donné à la chronique anonyme (RHC Occ., t. 3, p. 119-63). 
Cette interprétation s'imposera rapidement - et définitivement - dans le champ historiographique. Pour Louis Bréhier, l'éditeur et le traducteur français des Gesta, «par ses qualités de précision et de sincérité, le récit de l'Anonyme est une des sources fondamentales de l'histoire de la croisade ${ }^{20} »$ :

Sa valeur historique dépasse le seul récit des épisodes de la croisade. Par l'abondance et le caractère pittoresque des détails, par la naïveté et la spontanéité de ses réflexions, L'Anonyme nous renseigne admirablement sur l'état matériel et moral des bandes de croisés. Une pareille précision, un si grand nombre de détails pittoresques donnent à ce texte une valeur littéraire d'autant plus marquée qu'elle n'a été nullement recherchée par son auteur. Par bonheur, cette éducation [celle de Guibert ou Baudri] faisait défaut à notre chevalier, et il s'est contenté d'écrire probablement comme il parlait, sans rechercher aucun effet ${ }^{21}$.

Comme on aura pu le noter, d'anonyme l'auteur devient l' « Anonyme », un auteur de plein droit qu'on a vite assimilé à un simple soldat, pas très cultivé, et qui aurait parlé au nom de ses compagnons de guerre, comme le montre l'emploi récurrent du «nous » dans le récit : un soldat d'Italie du Sud, probablement de descendance normande, ce qui expliquerait son attachement initial à Bohémond, qu'il appelle fréquemment dominus, avant que ce dernier n'abandonne l'expédition. Ainsi, ce récit simple et naïf ne pourrait être le fait que d'un homme simple et naïf, sans grande envergure : un soldat de rang moyen, dont la parole aurait d'autant plus de valeur et d'authenticité qu'elle exprimerait aussi celle de milliers d'autres soldats qui formèrent le plus gros des troupes. Si l'auteur anonyme, rebaptisé l' «Anonyme », semble s'effacer devant les faits, il est en même temps considéré comme le porte-parole de toute une classe de guerriers, ceux dont on n'entend généralement guère la voix.

Le fait que son auteur ait été imaginé comme un soldat de rang moyen a accru la confiance en son témoignage : on fait sans doute plus confiance à un représentant de la « classe moyenne » (Bréhier) qu'à un clerc de haute volée, car on estime que les intérêts et les enjeux idéologiques étaient moindres pour le premier. Il n'avait pas à défendre obstinément l'Église, comme on l'attendrait d'un dignitaire ecclésiastique, ni la conduite des opérations, comme on l'attendrait de la part d'un chef militaire. La position «moyenne» de l'auteur renforce le caractère vrai, « sincère », de sa parole, qui rappelait à Rosalind Hill les lettres que son frère lui écrivait d'Afrique du Nord, pendant la Seconde Guerre Mondiale, en 1942-1943 ${ }^{22}$.

Le « style extrêmement concis, simple et sans ornement ${ }^{23}$ » du texte, sa structure très syncopée, essentiellement paratactique, juxtaposant les épisodes

20. L. BRÉHIER, op. cit., p. Xvii.

21. Ibid., p. xviii-xx.

22. Gesta Francorum et aliorum Hierosolimitanorum, R. HiLl éd., op. cit., p. xvii.

23. Ibid., p. xiv. 
les uns à la suite des autres, l'empathie de son auteur pour les faits et gestes des croisés, au nom desquels il s'exprimerait, tout cela a concouru à en faire le récit « normal » de la Première Croisade, la base indispensable de toute reconstitution de l'expédition qui mena à la fameuse conquête de Jérusalem en 1099.

Lire les Gesta, à en croire les historiens, c'est presque «être sur le terrain »; c'est pouvoir écouter les acteurs, percevoir au plus près les faits. Le texte doit ainsi se concevoir comme le miroir d'une réalité que l'on peut effleurer du doigt. L'auteur est un scribe, il n'est pas un écrivain ; il copie, il retranscrit ce qu'il voit, ce qu'il entend, il ne s'insinue pas entre les faits dont il a été témoin et qu'il décrit avec simplicité, avec authenticité. Avec les Gesta, nous nous approchons du récit «pur » imaginé par Benveniste, un récit qui rapporterait les événements tels qu'ils se sont déroulés, où le narrateur disparaît et où « les événements sont posés comme ils se sont produits à mesure qu'ils apparaissent à l'horizon de l'histoire », au point de donner au lecteur l'impression que «personne ne parle ici ; les événements semblent se raconter eux-mêmes ${ }^{24}$ ». Autrement dit, le « récit à l'état pur », comme le rappelle Gérard Genette, «c'est l'histoire elle-même, par opposition au récit pris en charge par le narrateur ${ }^{25} »$. Avec les Gesta, en quelque sorte, c'est comme si nous pouvions atteindre l'histoire avant qu'elle ne devienne récit, observer les faits avant leur transformation en discours.

Comme si... Comme si les Gesta, quel que soit le degré de simplicité, de naïveté du style, n'étaient pas, comme tout récit, comme toute œuvre littéraire, un objet construit, un discours soigneusement élaboré. En outre, comme l'ont déjà remarqué Hans Oehler et Colin Morris ${ }^{26}$, une analyse plus attentive révèle une image bien plus fine du texte et de son auteur. Certaines caractéristiques distinctives des Gesta montrent, en effet, une maîtrise consciente de procédés littéraires qui apportent au texte une coloration stylistique particulière. Notons d'abord la fréquente utilisation du cursus rythmé, c'est-à-dire l'ordonnancement contrôlé de syllabes finales d'une phrase, avec pour effet de rythmer les séquences narratives. Plus important encore semble l'usage symptomatique d'assonances et d'allitérations en de nombreux passages du texte ${ }^{27}$. Cet usage contrôlé de la

24. E. Benveniste, Problèmes de linguistique générale I, Paris, 1966, p. 241.

25. G. Genette, Figures II, Paris, 1972, p. 71.

26. H. Oehler, « Studien zu den Gesta Francorum », Mittellateinisches Jahrbuch, t. 6, 1970, p. 58-97 ; C. Morris, loc. cit.

27. Comme l'atteste ce passage que nous laissons sciemment en latin, et dans lequel les sons um et $u r$ s'enchaînent: "In die autem Ascensionis Domini coepimus urbem circumquaque inuadere, et aedificare instrumenta lignorum atque turres ligneas, quo possemus murales turres sternere. Tam fortiter et tam acriter aggredimur urbem per duos dies, ut etiam foderemus murum urbis. Turci quippe qui erant in urbe miserunt nuntios aliis qui uenerant adiutorium ciuitate dare ». La répétition du mot $u r b s$ est ici très significative, et c'est seulement après avoir à trois reprises usé d'allitérations, que l'auteur finit par le remplacer par civitas. 
langue est aussi perceptible dans les nombreux discours directs (dialogues ou proclamations) qui émaillent le texte, notamment dans ceux qui mettent en scène les chefs musulmans. C'est un latin pour le moins bariolé et hyperbolique qui est ainsi mis dans la bouche des ennemis des croisés. Notons à cet égard que Bréhier, à qui n'avait pas échappé cet aspect pour le moins singulier du texte, suggéra que le langage « fleuri » des musulmans ne pouvait appartenir au texte « original » mais qu'il était dû à un interpolateur ultérieur, parce que cela ne collait guère avec sa représentation d'un simple soldat au latin rudimentaire - il ne fallait pas risquer de compromettre le mythe...

Ce sont enfin les nombreuses références bibliques distillées tout au long du récit ${ }^{28}$ qui achèvent de nuancer fortement, voire de contredire, le portrait de l'auteur, de l' « Anonyme », tel qu'il a été traditionnellement brossé : plutôt qu'un simple soldat, il faudrait imaginer un clerc, certes pas de la pointure de Guibert de Nogent ou de Robert le Moine, mais un clerc tout de même, dont la maîtrise du latin aurait permis une certaine créativité langagière, dont les effets parfois appuyés (on pense notamment aux allitérations et rimes) suggèrent l'aspect délibérément oral, la dimension déclamatoire du texte destiné à être énoncé à haute voix. Les Gesta étaient donc certainement destinés à être lus en public. Leur latin élémentaire, leurs effets stylistiques pouvaient certainement toucher non seulement les clercs mais aussi la majorité des croisés de langue romane, qui devaient être capables de suivre un latin simple.

Cet exemple sert à nous rappeler, si besoin est, que le récit, aussi simple, aussi « cru » soit-il (tout au moins en apparence), est toujours un discours, une création individuelle, et qu'il doit être conçu comme un filtre par rapport aux « faits », à la réalité qu'il est censé, même le plus fidèlement, même le plus naïvement, rapporter. Ce bref rappel pourra faire sourire certains. Cela semble pourtant avoir échappé à plusieurs générations d'historiens soucieux de raconter la Première Croisade comme elle s'était « réellement » passée.

Non contents de trouver dans les Gesta une source des plus authentiques, plusieurs historiens ont même émis l'hypothèse que le texte n'était en fait que la réécriture d'une parole première, et qui aurait servi de source à la fois aux Gesta et à l'histoire de Pierre Tudebode - un Ur-text (le mot à lui seul contient sa part de mystère), un texte encore plus fruste, composé juste après la conquête de Jérusalem. À l'origine, se trouverait donc un autre récit qui nous rapprocherait encore un peu plus des événements, dont la reprise par l'auteur des Gesta et Pierre Tudebode constituerait les seules traces restantes. Un mythe se perpétuant et s'entretenant de lui-même, on a pu gloser à l'infini sur la nature de ce Ur-text dont on ne sait strictement rien, à commencer par son existence même. Un titre, « Histoire de Jérusalem », et quelques caractéristiques majeures

28. Comme le souligne bien Luigi Russo dans l'introduction de sa traduction italienne des Gesta (Le Gesta dei Franchi e degli altri pellegrini gerosolimitani, Alessandria, 2003, p. 9-12). 
lui ont même été attribués : «[le texte] manquait d'informations essentielles sur le début ou la fin de la croisade, et n'était guère cohérent ${ }^{29}{ }^{\prime}$. Il se pourrait bien, comme le dit John France, que l'hypothèse d'une nouvelle source soit tout simplement « un fantasme ${ }^{30} »$. Le vrai problème ne réside pas là. Ce qui pose problème, en effet, n'est pas tant la validité de l'argument proposé que la façon dont, une fois encore, la critique des sources se résume pour l'essentiel à une simple question de généalogie textuelle : les arguments tournent autour d'un hypothétique stemma au sommet duquel se trouverait la source originelle, encore saturée des clameurs et des souvenirs des soldats ${ }^{31}$.

Relater, encore et encore, les faits accomplis par les participants de la Croisade, suivre leurs pérégrinations quotidiennes, leurs souffrances, leurs obstacles, leurs succès, etc., c'est entretenir le mythe créé autour des Gesta: celui de croire que l'on pourra, un jour, restituer les faits dans leur "pureté » originelle. Ce mythe, qui s'est perpétué depuis nos auteurs médiévaux, a encore, si l'on en croit les Histoires des Croisades qui se succèdent à un rythme soutenu, un bel avenir devant lui. Mais de nouvelles voix se font depuis peu entendre, des voix qui entendent bien déterritorialiser (terme certes rébarbatif, mais ô combien adapté) les sources qui furent pendant longtemps l'apanage exclusif des spécialistes de la période, afin de les étudier non plus en tant que simple réservoir d'informations, mais en s'attachant davantage à explorer le contexte et les circonstances de leur production, leurs propriétés littéraires, leur structure narrative. Gageons donc que, bientôt, nous parlerons moins de res gestae, de faits, et plus d'historiae rerum gestarum, des textes narratifs grâce auxquels nous avons accès à ce passé, et dont seule une étude critique permettra de mettre en valeur et de contraster les différentes logiques politiques, sociales et culturelles à l'œuvre dans cet immense et singulier chantier textuel que fut la Première Croisade.

Damien KEMPF - School of History, University of Liverpool, 9 Abercromby Square, Liverpool L69 7WZ, Royaume-Uni

Marcus BULL - Department of History, University of North Carolina at Chapel Hill, Hamilton Hall, Chapel Hill, NC 27599-3195, États-Unis

29. J. Rubenstein, « What is the Gesta Francorum, and Who was Peter Tudebode », loc. cit., p. 202.

30. J. France, « The Use of the Anonymous Gesta Francorum », loc. cit. De façon significative, John France conclut son article en disant que si nous devons considérer les textes dérivés des Gesta comme des récits de plein droit, c'est parce que les informations supplémentaires qu'ils contiennent « pourraient être plus crédibles qu'on l'a supposé ». Une fois encore, quand l'attention se porte sur les différentes sources narratives de la Première Croisade, c'est pour mieux en extraire le sérum de vérité.

31. Pour un point de vue d'ensemble critique, et une lecture différente, des Gesta, voir l'introduction de la nouvelle édition des Gesta par M. Bull aux Presses d'Oxford ( $c f$. note 4). 